\title{
Hankel Determinant for $p$-Valent Alpha-Convex Functions
}

\author{
Gagandeep Singh and B. S. Mehrok \\ Department of Mathematics, M.S.K. Girls College, Bharowal (Tarn-Taran), Punjab 143401, India \\ Correspondence should be addressed to Gagandeep Singh; kamboj.gagandeep@yahoo.in
}

Received 14 June 2013; Accepted 29 August 2013

Academic Editor: Reza Saadati

Copyright (c) 2013 G. Singh and B. S. Mehrok. This is an open access article distributed under the Creative Commons Attribution License, which permits unrestricted use, distribution, and reproduction in any medium, provided the original work is properly cited.

The objective of the present paper is to obtain the sharp upper bound of $\left|a_{p+1} a_{p+3}-a_{p+2}^{2}\right|$ for $p$-valent $\alpha$-convex functions of the form $f(z)=z^{p}+\sum_{k=p+1}^{\infty} a_{k} z^{k}$ in the unit $\operatorname{disc} E=\{z:|z|<1\}$.

\section{Introduction}

Let $A_{p}$ be the class of analytic functions of the form

$$
f(z)=z^{p}+\sum_{k=p+1}^{\infty} a_{k} z^{k}
$$

in the unit $\operatorname{disc} E=\{z:|z|<1\}$ with $p \in N=\{1,2,3, \ldots\}$. Let $S$ be the subclass of $A_{1}=A$, consisting of univalent functions.

$S_{p}^{*}$ is the class consisting of functions of the form (1) and satisfying the condition

$$
\operatorname{Re}\left\{\frac{z f^{\prime}(z)}{p f(z)}\right\}>0, \quad z \in E .
$$

The functions of the class $S_{p}^{*}$ are called $p$-valent starlike functions. In particular, $S_{1}^{*} \equiv S^{*}$, the class of starlike functions.

$K_{p}$ is the class of functions of the form (1), satisfying the condition

$$
\operatorname{Re}\left\{\frac{\left(z f^{\prime}(z)\right)^{\prime}}{p f^{\prime}(z)}\right\}>0, \quad z \in E
$$

The functions of the class $K_{p}$ are known as $p$-valent convex functions. Particularly, $K_{1} \equiv K$, the class of convex functions.

Obviously $f(z) \in K_{p}$ if and only if $z f^{\prime}(z) / p \in S_{p}^{*}$.
Let $M_{p}(\alpha)(\alpha \geq 0)$ be the class of functions of the form (1), satisfying the condition

$$
\operatorname{Re}\left\{(1-\alpha) \frac{z f^{\prime}(z)}{p f(z)}+\alpha \frac{\left(z f^{\prime}(z)\right)^{\prime}}{p f^{\prime}(z)}\right\}>0, \quad z \in E .
$$

Functions in the class $M_{p}(\alpha)$ are known as $p$-valent alpha-convex functions. For $p=1$, the class $M_{p}(\alpha)$ reduces to the class $M(\alpha)$ of alpha-convex functions introduced by Mocanu [1]. Also $M_{p}(0) \equiv S_{p}^{*}$ and $M_{p}(1) \equiv K_{p}$.

In 1976, Noonan and Thomas [2] stated the qth Hankel determinant for $q \geq 1$ and $n \geq 1$ as

$$
H_{q}(n)=\left|\begin{array}{cccc}
a_{n} & a_{n+1} & \cdots & a_{n+q-1} \\
a_{n+1} & \cdots & \cdots & \cdots \\
\cdots & \cdots & \cdots & \cdots \\
a_{n+q-1} & \cdots & \cdots & a_{n+2 q-2}
\end{array}\right| .
$$

This determinant has also been considered by several authors. For example, Noor [3] determined the rate of growth of $H_{q}(n)$ as $n \rightarrow \infty$ for functions given by (1) with bounded boundary. Ehrenborg [4] studied the Hankel determinant of exponential polynomials. Also Hankel determinant was studied by various authors including Hayman [5] and Pommerenke [6]. In [7], Janteng et al. studied the Hankel determinant for the classes of starlike and convex functions. Again Janteng et al. discussed the Hankel determinant problem for the classes of starlike functions with respect to symmetric points and convex functions with respect to symmetric 
points in [8] and for the functions whose derivative has a positive real part in [9]. Also Hankel determinant for various subclasses of $p$-valent functions was investigated by various authors including Krishna and Ramreddy [10] and Hayami and Owa [11].

Easily, one can observe that the Fekete and Szegö functional is $\mathrm{H}_{2}(1)$. Fekete and Szegö [12] then further generalised the estimate $\left|a_{3}-\mu a_{2}^{2}\right|$, where $\mu$ is real and $f \in S$. For our discussion in this paper, we consider the Hankel determinant in the case of $q=2$ and $n=2$ :

$$
\left|\begin{array}{ll}
a_{2} & a_{3} \\
a_{3} & a_{4}
\end{array}\right|
$$

In this paper, we seek sharp upper bound of the functional $\left|a_{p+1} a_{p+3}-a_{p+2}^{2}\right|$ for functions belonging to the class $M_{p}(\alpha)$. The results due to Janteng et al. [7] follow as special cases.

\section{Preliminary Results}

Let $Q$ be the family of all functions $q$ analytic in $E$ for which $\operatorname{Re}(q(z))>0$ and

$$
q(z)=1+q_{1} z+q_{2} z^{2}+\cdots
$$

for $z \in E$.

Lemma 1 (see [6]). If $q \in Q$, then $\left|q_{k}\right| \leq 2(k=1,2,3, \ldots)$.

Lemma 2 (see $[13,14])$. If $q \in Q$, then

$$
\begin{aligned}
2 q_{2}= & q_{1}^{2}+\left(4-q_{1}^{2}\right) x, \\
4 q_{3}= & q_{1}^{3}+2 q_{1}\left(4-q_{1}^{2}\right) x-q_{1}\left(4-q_{1}^{2}\right) x^{2} \\
& +2\left(4-q_{1}^{2}\right)\left(1-|x|^{2}\right) z
\end{aligned}
$$

for some $x$ and $z$ satisfying $|x| \leq 1,|z| \leq 1$ and $q_{1} \in[0,2]$.

\section{Main Result}

Theorem 3. If $f \in M_{p}(\alpha)$, then

$$
\left|a_{p+1} a_{p+3}-a_{p+2}^{2}\right| \leq \frac{p^{4}}{(p+2 \alpha)^{2}}\left[1-\frac{12 \alpha^{2}(p+\alpha)^{4}}{(p+3 \alpha) A(\alpha)}\right],
$$

where

$$
\begin{aligned}
A(\alpha)= & -24 p(p+2 \alpha)\left[\alpha(p+1)(p+2)-(\alpha-1) p^{2}\right] \\
& \times\left[(p+\alpha)^{2}+p\left(p^{2}+\alpha+2 \alpha p\right)\right] \\
& +12 p(p+3 \alpha)\left(p^{2}+\alpha+2 \alpha p\right) \\
& \times\left[p\left(p^{2}+\alpha+2 \alpha p\right)+2(p+\alpha)^{2}\right] \\
& +4(p+\alpha)^{3}\left[3(p+\alpha)(p+3 \alpha)-4(p+2 \alpha)^{2}\right] \\
& -16 p^{2}(p+2 \alpha)^{2}\left[(\alpha-1) p^{3}-\alpha(p+1)^{3}\right] .
\end{aligned}
$$

Proof. Since $f \in M_{p}(\alpha)$, so from (4)

$$
(1-\alpha) \frac{z f^{\prime}(z)}{p f(z)}+\alpha \frac{\left(z f^{\prime}(z)\right)^{\prime}}{p f^{\prime}(z)}=q(z)
$$

On expanding and equating the coefficients of $z, z^{2}$, and $z^{3}$ in (11), we get

$$
\begin{gathered}
a_{p+1}=\frac{p^{2} q_{1}}{p+\alpha} \\
a_{p+2}=\frac{p^{2} q_{2}}{2(p+2 \alpha)}+\frac{p^{3}\left(p^{2}+\alpha+2 \alpha p\right) q_{1}^{2}}{2(p+2 \alpha)(p+\alpha)^{2}}, \\
a_{p+3}=\frac{p^{2} q_{3}}{3(p+3 \alpha)} \\
+\frac{\left[3 \alpha(p+1)(p+2)-3(\alpha-1) p^{2}\right] p^{3} q_{1} q_{2}}{6(p+\alpha)(p+2 \alpha)(p+3 \alpha)} \\
+p^{4}\left[\left(p^{2}+\alpha+2 \alpha p\right)\right. \\
\times\left(3 \alpha(p+1)(p+2)-3(\alpha-1) p^{2}\right) \\
\quad \times\left(6(p+2 \alpha)(p+3 \alpha)(p+\alpha)^{3}\right)^{-1} .
\end{gathered}
$$

Equation (12) yields:

$$
\begin{aligned}
& a_{p+1} a_{p+3}-a_{p+2}^{2} \\
& =\frac{p^{4}}{C(\alpha)} \\
& \times\left\{4(p+2 \alpha)^{2}(p+\alpha)^{3} q_{1} q_{3}\right. \\
& +\left[2 p(p+2 \alpha)(p+\alpha)^{2}\right. \\
& \quad \times\left(3 \alpha(p+1)(p+2)-3(\alpha-1) p^{2}\right) \\
& \quad-6 p(p+3 \alpha)(p+\alpha)^{2} \\
& \left.\quad \times\left(p^{2}+\alpha+2 \alpha p\right)\right] q_{1}^{2} q_{2} \\
& +\left[2 p^{2}(p+2 \alpha)\right. \\
& \quad \times\left(\left(p^{2}+\alpha+2 \alpha p\right)\right. \\
& \quad \times\left(3 \alpha(p+1)(p+2)-3(\alpha-1) p^{2}\right) \\
& \left.+2(p+2 \alpha)\left((\alpha-1) p^{3}-\alpha(p+1)^{3}\right)\right)
\end{aligned}
$$




$$
\begin{aligned}
& \left.-3 p^{2}(p+3 \alpha)\left(p^{2}+\alpha+2 \alpha p\right)^{2}\right] q_{1}^{4} \\
& \left.-3(p+3 \alpha)(p+\alpha)^{4} q_{2}^{2}\right\},
\end{aligned}
$$

where $C(\alpha)=12(p+3 \alpha)(p+2 \alpha)^{2}(p+\alpha)^{4}$.

Using Lemmas 1 and 2 in (13), we obtain

$$
\begin{aligned}
& \left|a_{p+1} a_{p+3}-a_{p+2}^{2}\right| \\
& =\frac{p^{4}}{4 C(\alpha)} \\
& \times \mid-\left[-4(p+2 \alpha)^{2}(p+\alpha)^{3}+3(p+\alpha)^{4}\right. \\
& \times(p+3 \alpha)-4 p(p+2 \alpha)(p+\alpha)^{2} \\
& \times\left(3 \alpha(p+1)(p+2)-3(\alpha-1) p^{2}\right) \\
& +12 p(p+3 \alpha)(p+\alpha)^{2}\left(p^{2}+\alpha+2 \alpha p\right) \\
& +12 p^{2}(p+3 \alpha)\left(p^{2}+\alpha+2 \alpha p\right)^{2} \\
& -8 p^{2}(p+2 \alpha)\left(p^{2}+\alpha+2 \alpha p\right) \\
& \times\left[3 \alpha(p+1)(p+2)-3(\alpha-1) p^{2}\right] \\
& \left.-16 p^{2}(p+2 \alpha)^{2}\left[(\alpha-1) p^{3}-\alpha(p+1)^{3}\right]\right] q_{1}^{4} \\
& +(p+\alpha)^{2}\left[8(p+2 \alpha)^{2}(p+\alpha)+4 p(p+2 \alpha)\right. \\
& \times\left(3 \alpha(p+1)(p+2)-3(\alpha-1) p^{2}\right) \\
& -6(p+\alpha)^{2}(p+3 \alpha)-12 p(p+3 \alpha) \\
& \left.\times\left(p^{2}+\alpha+2 \alpha p\right)\right] q_{1}^{2}\left(4-q_{1}^{2}\right) x \\
& -(p+\alpha)^{3}\left[4(p+2 \alpha)^{2} q_{1}^{2}+3(p+\alpha)(p+3 \alpha)\right. \\
& \left.\times\left(4-q_{1}^{2}\right)\right]\left(4-q_{1}^{2}\right) x^{2} \\
& +8(p+\alpha)^{3}(p+2 \alpha)^{2} q_{1}\left(4-q_{1}^{2}\right)\left(1-|x|^{2}\right) z \mid .
\end{aligned}
$$

Assume that $q_{1}=q$ and $q \in[0,2]$; using triangular inequality and $|z| \leq 1$, we have

$$
\begin{aligned}
& \left|a_{p+1} a_{p+3}-a_{p+2}^{2}\right| \\
& \leq \frac{p^{4}}{4 C(\alpha)} \\
& \times\left\{\left[-4(p+2 \alpha)^{2}(p+\alpha)^{3}+3(p+\alpha)^{4}\right.\right. \\
& \times(p+3 \alpha)-4 p(p+2 \alpha)(p+\alpha)^{2} \\
& \times\left(3 \alpha(p+1)(p+2)-3(\alpha-1) p^{2}\right)
\end{aligned}
$$

$$
\begin{gathered}
+12 p(p+3 \alpha)(p+\alpha)^{2}\left(p^{2}+\alpha+2 \alpha p\right) \\
+12 p^{2}(p+3 \alpha)\left(p^{2}+\alpha+2 \alpha p\right)^{2} \\
-8 p^{2}(p+2 \alpha)\left(p^{2}+\alpha+2 \alpha p\right) \\
\times\left[3 \alpha(p+1)(p+2)-3(\alpha-1) p^{2}\right] \\
\left.-16 p^{2}(p+2 \alpha)^{2}\left[(\alpha-1) p^{3}-\alpha(p+1)^{3}\right]\right] q^{4} \\
+(p+\alpha)^{2}\left[8(p+2 \alpha)^{2}(p+\alpha)+4 p(p+2 \alpha)\right. \\
\times\left(3 \alpha(p+1)(p+2)-3(\alpha-1) p^{2}\right) \\
-6(p+\alpha)^{2}(p+3 \alpha)-12 p(p+3 \alpha) \\
\left.\times\left(p^{2}+\alpha+2 \alpha p\right)\right] q^{2}\left(4-q^{2}\right) \delta \\
+8(p+\alpha)^{3}(p+2 \alpha)^{2} q\left(4-q^{2}\right) \\
+(p+\alpha)^{3}\left[\left(4(p+2 \alpha)^{2}-3(p+\alpha)(p+3 \alpha)\right) q^{2}\right. \\
-8(p+2 \alpha)^{2} q+12(p+\alpha) \\
\left.\times(p+3 \alpha)]\left(4-q^{2}\right) \delta^{2}\right\} \\
=\frac{p^{4}}{4 C(\alpha)} F(\delta), \quad \text { where } \delta=|x| \leq 1 .
\end{gathered}
$$

It is easy to verify that $F(\delta)$ is an increasing function and so Max. $F(\delta)=F(1)$.

Consequently

$$
\left|a_{p+1} a_{p+3}-a_{p+2}^{2}\right| \leq \frac{p^{4}}{4 C(\alpha)} G(q),
$$

where

$$
G(q)=F(1) .
$$

So

$$
G(q)=A(\alpha) q^{4}+48 \alpha(p+\alpha)^{4} q^{2}+48(p+3 \alpha)(p+\alpha)^{4},
$$

where $A(\alpha)$ is defined in (10).

Now

$$
\begin{aligned}
& G^{\prime}(q)=4 A(\alpha) q^{3}+96 \alpha(p+\alpha)^{4} q, \\
& G^{\prime \prime}(q)=12 A(\alpha) q^{2}+96 \alpha(p+\alpha)^{4} .
\end{aligned}
$$

$G^{\prime}(q)=0$ gives

$$
4 q\left[A(\alpha) q^{2}+24 \alpha(p+\alpha)^{4}\right]=0 .
$$

$G^{\prime \prime}(q)$ is negative at $q=\sqrt{-24 \alpha(p+\alpha)^{4} / A(\alpha)}=q^{\prime}$. 
So

$$
\operatorname{Max} \cdot G(q)=G\left(q^{\prime}\right) .
$$

Hence from (15), we obtain (9).

The result is sharp for $q_{1}=q^{\prime}, q_{2}=q_{1}^{2}-2$, and $q_{3}=$ $q_{1}\left(q_{1}^{2}-3\right)$.

For $\alpha=0$, Theorem 3 gives the following result.

Corollary 4. If $f(z) \in S_{p}^{*}$, then

$$
\left|a_{p+1} a_{p+3}-a_{p+2}^{2}\right| \leq p^{2} .
$$

For $\alpha=1$, Theorem 3 yields.

Corollary 5. If $f(z) \in K_{p}$, then

$$
\begin{aligned}
& \left|a_{p+1} a_{p+3}-a_{p+2}^{2}\right| \\
& \quad \leq \frac{p^{4}}{(p+2)^{2}}\left[1+\frac{3}{(p+3)\left(-p^{3}-3 p^{2}+3 p+7\right)}\right] .
\end{aligned}
$$

Putting $\alpha=0$ and $p=1$ in Theorem 3, we obtain the following result due to Janteng et al. [7].

Corollary 6. If $f(z) \in S^{*}$, then

$$
\left|a_{2} a_{4}-a_{3}^{2}\right| \leq 1 \text {. }
$$

Putting $\alpha=1$ and $p=1$ in Theorem 3, we obtain the following result due to Janteng et al. [7].

Corollary 7. If $f(z) \in K$, then

$$
\left|a_{2} a_{4}-a_{3}^{2}\right| \leq \frac{1}{8}
$$

\section{References}

[1] P. T. Mocanu, "Une propriété de convexité généralisée dans la théorie de la représentation conforme," Mathematica, vol. 11, no. 34, pp. 127-133, 1969.

[2] J. W. Noonan and D. K. Thomas, "On the second Hankel determinant of areally mean $p$-valent functions," Transactions of the American Mathematical Society, vol. 223, pp. 337-346, 1976.

[3] K. I. Noor, "Hankel determinant problem for the class of functions with bounded boundary rotation," Académie de la République Populaire Roumaine. Revue Roumaine de Mathématiques Pures et Appliquées, vol. 28, no. 8, pp. 731-739, 1983.

[4] R. Ehrenborg, "The Hankel determinant of exponential polynomials," The American Mathematical Monthly, vol. 107, no. 6, pp. 557-560, 2000.

[5] W. K. Hayman, Multivalent Functions, vol. 48 of Cambridge Tracts in Mathematics and Mathematical Physics, Cambridge University Press, Cambridge, UK, 1958.

[6] Ch. Pommerenke, Univalent Functions, Vandenhoeck \& Ruprecht, Göttingen, 1975.

[7] A. Janteng, S. A. Halim, and M. Darus, "Hankel determinant for starlike and convex functions," International Journal of Mathematical Analysis, vol. 1, no. 13-16, pp. 619-625, 2007.
[8] A. Janteng, S. A. Halim, and M. Darus, "Hankel determinant for functions starlike and convex with respect to symmetric points," Journal of Quality Measurement and Analysis, vol. 2, no. 1, pp. 37-43, 2006.

[9] A. Janteng, S. A. Halim, and M. Darus, "Coefficient inequality for a function whose derivative has a positive real part," Journal of Inequalities in Pure and Applied Mathematics, vol. 7, no. 2, article 50, 2006.

[10] D. V. Krishna and T. Ramreddy, "Hankel determinant for $p$ valent starlike and convex functions of order $\alpha$," Novi Sad Journal of Mathematics, vol. 42, no. 2, pp. 89-96, 2012.

[11] T. Hayami and S. Owa, "Hankel determinant for $p$-valently starlike and convex functions of order $\alpha$," General Mathematics, vol. 17, no. 4, pp. 29-44, 2009.

[12] M. Fekete and G. Szegö, "Eine Bemerkung über ungerade schlichte Funktionen," Journal of the London Mathematical Society, vol. 8, pp. 85-89, 1933.

[13] R. J. Libera and E. J. Złotkiewicz, "Early coefficients of the inverse of a regular convex function," Proceedings of the American Mathematical Society, vol. 85, no. 2, pp. 225-230, 1982.

[14] R. J. Libera and E. J. Złotkiewicz, "Coefficient bounds for the inverse of a function with derivative in P," Proceedings of the American Mathematical Society, vol. 87, no. 2, pp. 251-257, 1983. 


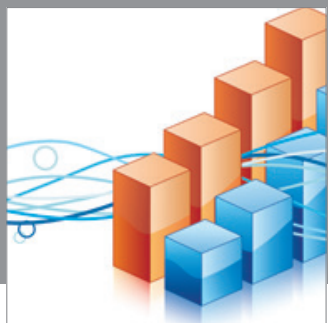

Advances in

Operations Research

mansans

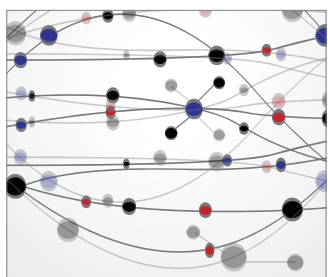

The Scientific World Journal
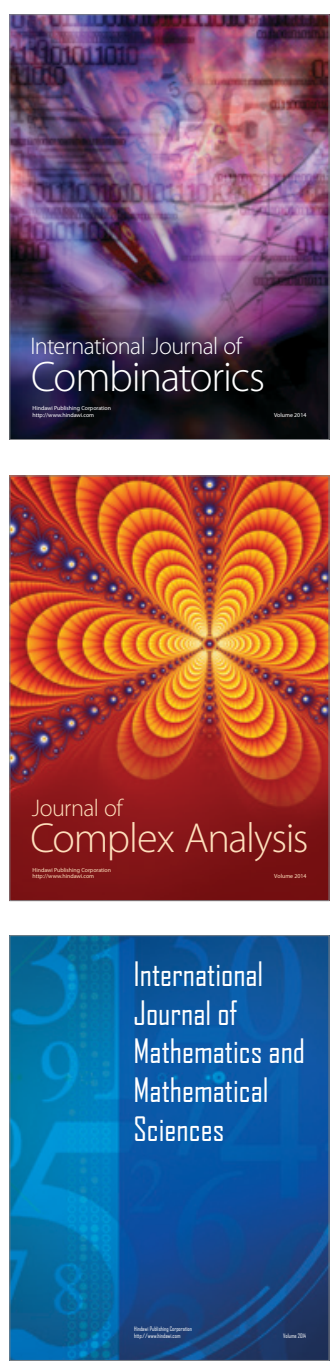
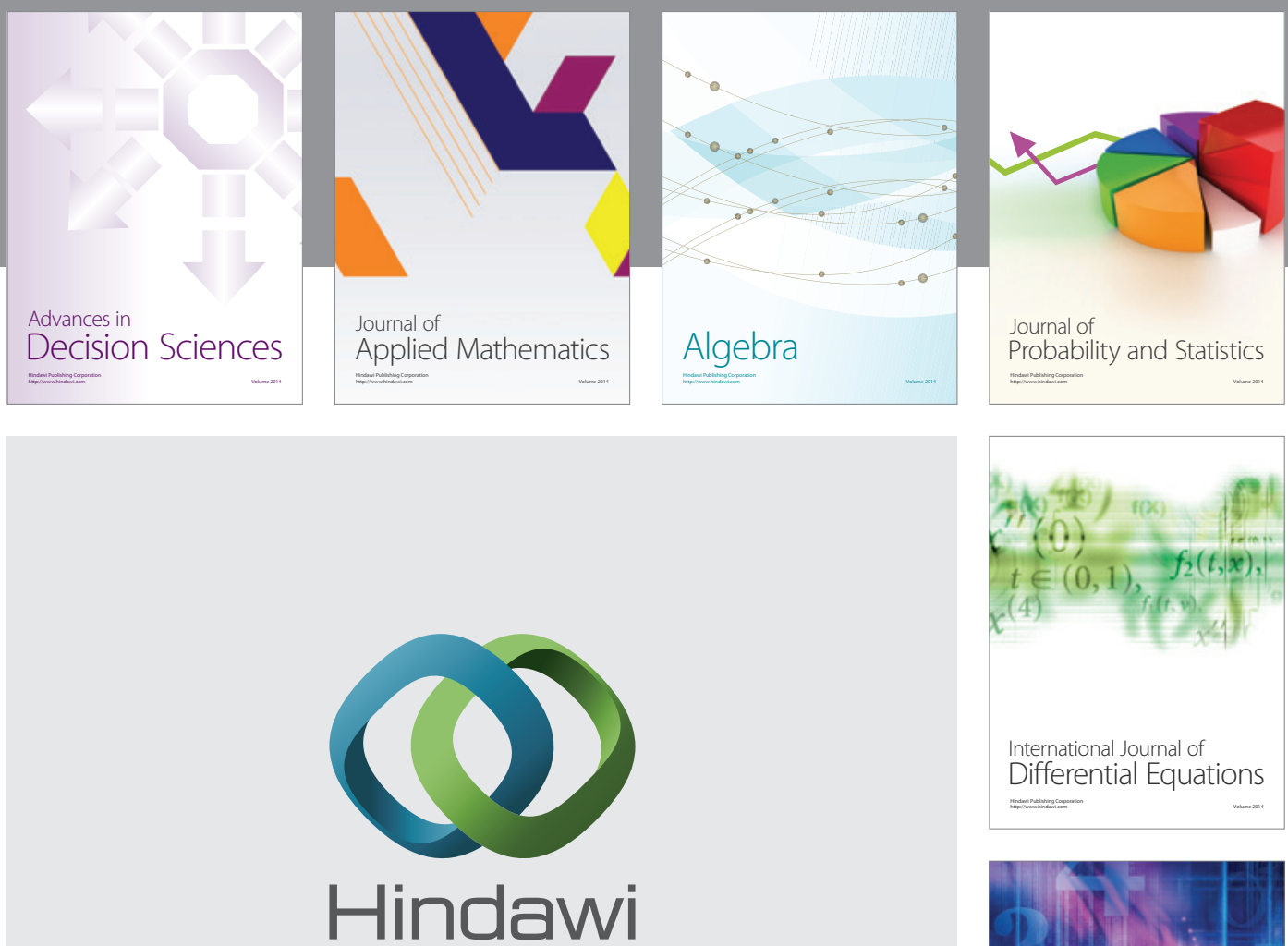

Submit your manuscripts at http://www.hindawi.com
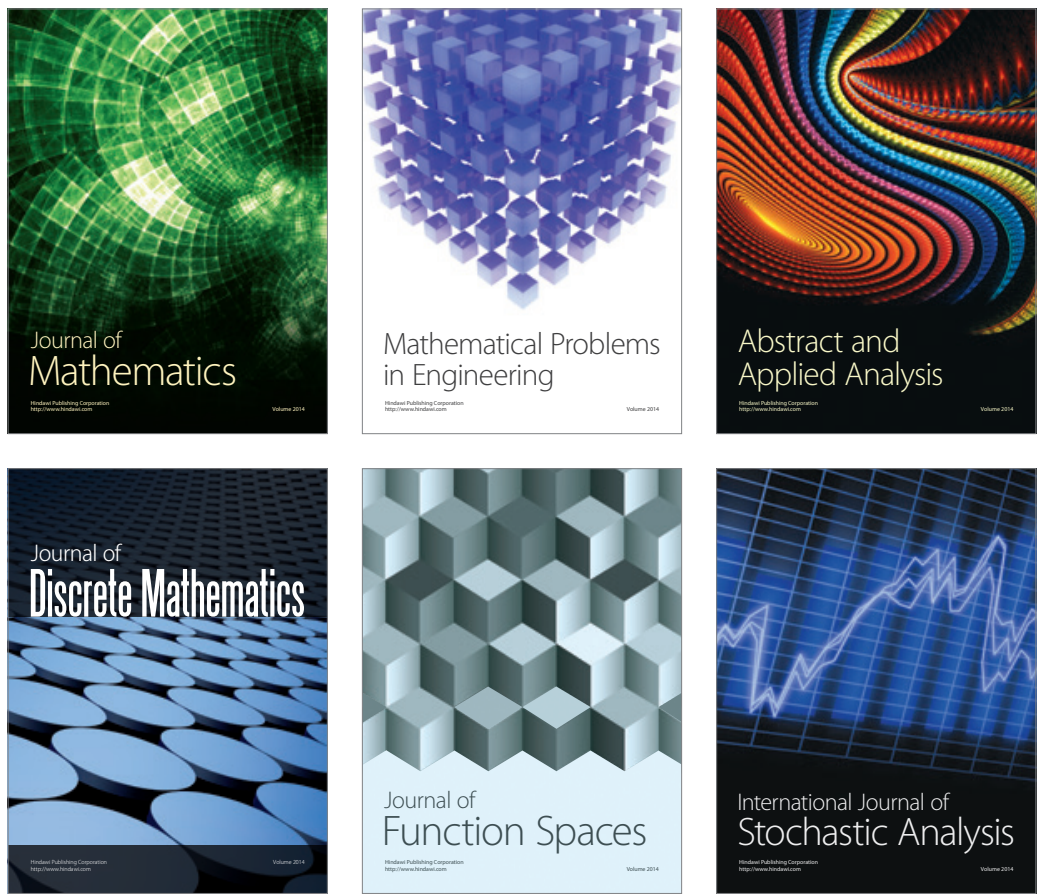

Journal of

Function Spaces

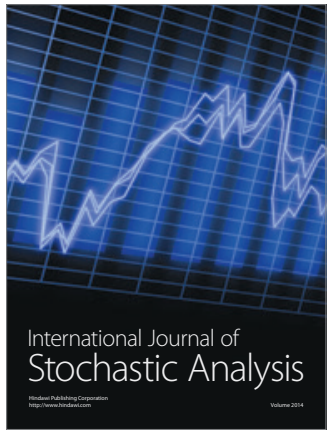

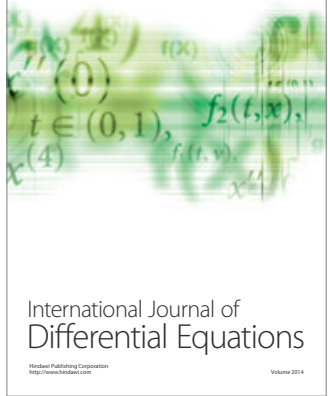
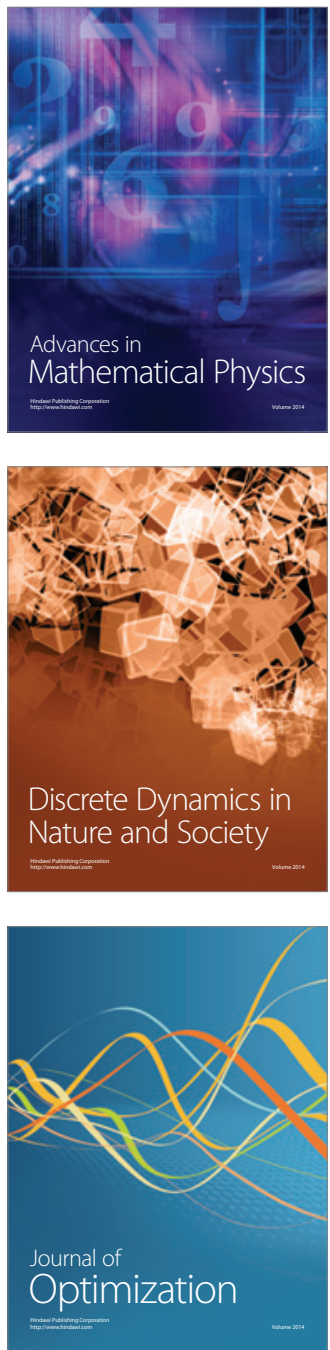